\title{
COMUNICAÇÃO
}

\section{USO DE REGULADORES VEGETAIS NA CONSERVAÇÃO REFRIGERADA DE ACEROLAS (Malpighia glabra L.)}

\author{
Effect of plant growth regulators in the refrigerated conservation of acerola fruits \\ (Malpighia glabra L.)
}

\author{
Andréa Maria Antunes ${ }^{1}$, Janice Valmórbida ${ }^{2}$, Elizabeth Orika Ono ${ }^{3}$, João Domingos Rodrigues ${ }^{4}$
}

\begin{abstract}
RESUMO
Com o objetivo de avaliar o uso do ácido giberélico $\left(\mathrm{GA}_{3}\right)$ e da benzilaminopurina (BAP) na conservação de acerolas (Malpighia glabra L.) colhidas no estádio verde e armazenadas sob refrigeração, acerolas foram submetidas aos seguintes tratamentos, sob imersão por 30 minutos: controle (água), $50 \mathrm{mg} \mathrm{L}^{-1}$ e $100 \mathrm{mg} \mathrm{L}^{-1} \mathrm{de} \mathrm{GA}_{3}, 50 \mathrm{mg} \mathrm{L}^{-1} \mathrm{e} 100 \mathrm{mg} \mathrm{L}^{-1}$ de BAP. Após os tratamentos, os frutos foram deixados para secar ao ar em local fresco e, então, embalados em bandejas de isopor cobertas com filme de polietileno e armazenados em câmara B.O.D a $8 \pm 1^{\circ} \mathrm{C}$, por 14 dias. As avaliações foram realizadas em intervalos de 4 dias. Os frutos amostrados foram submetidos a avaliações de coloração, teor de sólidos solúveis, acidez titulável e teor de ácido ascórbico. A análise dos resultados mostrou que a aplicação dos reguladores não teve efeito no aumento da conservação refrigerada de acerolas e que somente a refrigeração foi suficiente para conservá-las durante 14 dias.
\end{abstract}

Termos para indexação: Giberelina, citocinina, refrigeração, pós-colheita, Malpighia glabra.

\begin{abstract}
With the objective of preserving the physicochemical characteristics from green stage harvested of acerola fruits (Malpighia glabra L.), these were submitted to the following 30-minute immersion treatments: control (water), 50mg L $\mathrm{m}^{-1} \mathrm{GA}_{3}, 100 \mathrm{mg} \mathrm{L}^{-1} \mathrm{GA}_{3}$, $50 \mathrm{mg} \mathrm{L}^{-1}$ BAP (benzylaminopurine), and $100 \mathrm{mg} \mathrm{L}^{-1}$ BAP. After treatment, the fruits were air-dried in a cool place and then packaged in styrofoam trays wrapped with polyethylene film, and stored in a BOD incubator at $8^{\circ} \mathrm{C}$ for 14 days. Evaluations were performed at 4 days intervals. The fruits were evaluated for coloration, soluble solids content, trituratable acidity, and ascorbic acid content. The analysis of the results showed that the application of plant growth regulators did not have effect in the increase of the refrigerated conservation of acerola fruits and that only the cooling was enough to conserve its for 14 days.
\end{abstract}

Index terms: Gibberellin, cytokinin, refrigeration, post harvest, Malpighia glabra.

\section{(Recebido para publicação em 20 de outubro de 2005 e aprovado em 15 de maio de 2006)}

A aceroleira (Malpighia glabra L.) é uma frutífera cujos pequenos frutos vermelhos são semelhantes à cereja européia. É considerada uma das mais ricas fontes de vitamina $\mathrm{C}$, superando em várias vezes frutas como goiaba, caju, laranja e limão que são excelentes fontes dessa vitamina (ALVES et al., 1995; INSTITUTO BRASILEIRO DE FRUTAS, 1995).

A acerola tem despertado a atenção dos agricultores não somente por seu elevado conteúdo de vitamina C $(2.500$ a $4.500 \mathrm{mg} / 100 \mathrm{~g}$ de polpa) em relação a outras frutas, mas também por seu potencial para industrialização, uma vez que pode ser consumida sob forma de sucos, compotas, geléias, utilizada no enriquecimento de sucos e de alimentos dietéticos, na forma de alimentos nutracêuticos, como comprimidos ou cápsulas, empregados como suplemento alimentar, chás, bebidas para esportistas, barras nutritivas e iogurtes (PÍPOLO et al., 2002).

Nogueira et al. (2002) concluíram que frutos verdes apresentam teores de vitamina $\mathrm{C}$ mais elvados que os maduros e semimaduros, podendo ser utilizados pela indústria farmacêutica. O teor de ácido ascórbico de acerolas decresce com a evolução do amadurecimento (CRUZ et al., 1995). Frutos maduros com coloração vermelho intensa, apresentam elevado teor de açúcares, baixa acidez e menores teores de vitamina $\mathrm{C}$, ainda assim, superando cerca de 20 a 30 vezes o teor dos frutos cítricos

\footnotetext{
1Universidade Estadual Paulista/UNESP - Pós-Graduanda do Programa de Ciências Biológicas - Botânica - Departamento de Botânica - IB - Cx. P. 510 18618-000 - Botucatu, SP - andreamantunes@yahoo.com.br

2 Universidade Estadual Paulista/UNESP - Pós-Graduanda do Programa de Agronomia - AC: Horticultura - Cx. P. 237 - $18603-970$ - FCA/UNESP _ Botucatu, SP _ janice@fca.unesp.br

${ }^{3}$ Universidade Estadual Paulista/UNESP - Professora Adjunta/Livre - Docente - Departamento de Botânica - IB, Cx. P. 510 - $18618-000$ Botucatu, SP - eoono@ibb.unesp.br

${ }^{4}$ Universidade Estadual Paulista/UNESP - Departamento de Botânica - IB - Professor Titular - Cx. P. 510 - 18618-000 - Botucatu, SP mingo@ibb.unesp.br
} 
(ALVES et al., 1995). O fator determinante do ponto de colheita é o destino de uso dos frutos, que podem ser colhidos no início da maturação (verdes), quando destinados à fabricação de produtos em pó, cápsulas, concentrados para o enriquecimento de outros alimentos, sendo o teor de vitamina $\mathrm{C}$ é a característica mais importante (ALVES, 1996).

Petinari \& Tarsitano (2002) sugerem maiores estudos na conservação pós-colheita dos frutos de aceroleira, pois por falta de conservação muitos produtores têm produzido para mercado 'in natura' e não para indústria, gerando descontentamento, principalmente na época de maior produção.

$\mathrm{O}$ armazenamento refrigerado prolonga o período de comercialização da fruta fresca, porém não evita alterações físico-químicas, que depreciam sua qualidade (CARVALHO \& MANICA, 1994). Dentre as alternativas que visam minimizar estes problemas, destacam-se o uso de embalagens de polietileno, aplicação de cera e aplicação de reguladores vegetais, a fim de permitir melhor conservação dos frutos (CARVALHO, 1994).

De acordo com Chitarra \& Chitarra (1990), já foi evidenciado o papel das auxinas, giberelinas e citocininas no amadurecimento dos frutos, as quais têm sido estudadas como retardadores da senescência, enquanto que o etileno e ácido abscísico são tidos como promotores do amadurecimento em frutos. As giberelinas afetam na mudança de cor, uma vez que retardam a perda de clorofila, acúmulo de carotenóides e o amaciamento da casca.

Teaotia et al. (1972) relatam que o ácido giberélico, quando aplicado em pós-colheita, teve efeito retardador ao amadurecimento de goiabas, reduzindo a taxa de respiração e as mudanças na coloração.

A aplicação exógena de reguladores vegetais como a citocinina, tem sido utilizada na manutenção da coloração verde da casca do limão 'Tahiti' por um período de tempo maior e no retardo da senescência (FIORAVANÇO et al., 1995).

A acerola é um fruto climatérico e passa por uma série de alterações durante os processos de maturação, amadurecimento e senescência (ALVES, 1993), destacando-se degradação da clorofila, aparecimento dos carotenóides, decréscimo na acidez, acentuada perda de vitamina $\mathrm{C}$ ao longo dessas etapas. Estas podem acontecer quando o fruto está na planta ou após a colheita, podendo os frutos serem colhidos no início da maturação (verde, verde-amarelado ou até início da pigmentação vermelha), quando se destinam à produção de vitamina $\mathrm{C}$.

$\mathrm{O}$ retardo do amadurecimento é fundamental por manter maiores níveis de ácido ascóbico, assim, o trabalho objetivou avaliar o uso do $\mathrm{GA}_{3}$ e da benzilaminopurina na conservação de acerolas armazenadas sob refrigeração, como uma alternativa para manter os frutos verdes e viáveis por mais tempo, visando sua utilização na indústria farmacêutica.

Acerolas no estádio verde foram colhidas na região de Bauru (latitude 22 21'30" Sul, longitude 4901'37" Oeste), SP e transportadas ao Departamento de Gestão e Tecnologia Agroindustrial, FCA, UNESP - Botucatu -SP. Os frutos foram selecionados para eliminar aqueles com danos mecânicos ou doenças, lavados e sanificados com solução de hipoclorito de sódio a $0,25 \%$ por 15 minutos para desinfecção fúngica, enxaguados com água destilada e secos em papel de filtro.

Depois de secos, foram tratados com os reguladores vegetais, através de imersão por 30 minutos, secos ao ar e acondicionados em bandejas de isopor (250 x 150 x $25 \mathrm{~mm}$ ) cobertos por filme de policloreto de vinila (PVC) com 14 micras de espessura e armazenados em câmara B.O.D. a $8 \pm 1^{\circ} \mathrm{C}$. Os tratamentos utilizados foram: T1- controle- imersão em água; T2- imersão em $50 \mathrm{mg} \mathrm{L}^{-1}$ de GA 3 ; T3- imersão em $100 \mathrm{mg} \mathrm{L}^{-1}$ de $\mathrm{GA}_{3}$; T4- imersão em $50 \mathrm{mg} \mathrm{L}^{-1}$ de BAP e T5- imersão em $100 \mathrm{mg} \mathrm{L}^{-1}$ de BAP.

$\mathrm{O} \mathrm{GA}_{3}$ foi utilizado na forma do produto comercial Pro-Gibb contendo $10 \%$ de $\mathrm{GA}_{3}$ e a citocinina utilizada foi a benzilaminopurina (BAP), p.a. da Sigma Laboratórios.

Amostras de cada parcela foram retiradas ao acaso e submetidas às respectivas análises, no tempo zero de armazenamento para comparação e aos 2, 6, 10 e 14 dias de armazenamento.

As determinações efetuadas foram: observação visual da coloração da casca dos frutos, segundo escala subjetiva de valores, considerando frutos verdes, os que apresentavam mais de $75 \%$ da casca com coloração verde (pontuação 1); frutos semimaduros, aqueles com mais de $75 \%$ da casca com coloração amarelada (pontuação 2) e frutos maduros aqueles com mais de $75 \%$ da casca com coloração vermelha (pontuação 3); o teor de sólidos solúveis ( ${ }^{\circ}$ Brix) utilizando-se refratômetro digital Attago, cuja amostragem correspondeu a alíquota dos frutos triturados; a acidez titulável (AT) foi determinada por titulação do suco de acerola com solução de hidróxido de sódio $(\mathrm{NaOH}) 0,1 \mathrm{~N}$; teor de vitamina $\mathrm{C}$, conforme metodologia de Tillmmans (1927), citado por Bezerra Neto et al. (1994), sendo o resultado expresso em $\mathrm{mg}$ de ácido ascórbico por $100 \mathrm{~mL}$ da amostra.

$O$ delineamento experimental utilizado foi inteiramente casualizado, com 3 repetições e 56 frutos/ bandeja, num esquema fatorial $5 \mathrm{X} 4$ (correspondentes a 5 tratamentos- controle; $50 \mathrm{mg} \mathrm{L}^{-1} \mathrm{de} \mathrm{GA}_{3} ; 100 \mathrm{mg} \mathrm{L}^{-1} \mathrm{de}$ 
GA $50 \mathrm{mg} \mathrm{L}^{-1}$ de BAP; $100 \mathrm{mg} \mathrm{L}^{-1}$ de BAP e 4 tempos de armazenamento). Os resultados foram submetidos à análise de variância e as médias comparadas pelo teste Tukey a $5 \%$ de probabilidade.

Os resultados não revelaram diferenças significativas entre os tratamentos quanto à coloração. Todos os frutos receberam pontuação número 1 segundo a escala subjetiva de cores, permanecendo assim durante todas as avaliações.

Melo et al. (2000), avaliando a temperatura de armazenamento para pitanga, observaram que os frutos verdes sob refrigeração a $8^{\circ} \mathrm{C}$ apenas começaram a amarelar no quinto dia de armazenamento, não atingindo a cor vermelha até o final do experimento. Neste experimento, os frutos de acerola permaneceram com a mesma coloração durante os 14 dias, em todos os tratamentos, incluindo o controle. Pode-se atribuir esse resultado ao tratamento refrigerado a $8 \pm 1^{\circ} \mathrm{C}$, juntamente, com o auxílio do filme de PVC e ao tempo de armazenamento de apenas 14 dias. Fioravanço et al. (1995), usando citocinina e recobrimentos em limão 'Tahiti' armazenado em temperatura controlada, observaram que clorofilas "a" e "b" foram pouco influenciadas pela citocinina, enquanto os frutos tratados com cera e e embalagem com polietileno, conjuntamente ou isolados, à temperatura de 7,5 a $8,5^{\circ} \mathrm{C}$, tiveram o seu tempo de conservação prolongado. Isso porque a aplicação de ceras ou outros recobrimentos diminui trocas gasosas e inibe desenvolvimento da cor amarela, uma vez que $\mathrm{CO}_{2}$ é um inibidor competititvo da ação do etileno, cuja síntese também é inibida por baixos teores de $\mathrm{O}_{2}$ (AHRENS \& BARMORE, 1988). O armazenamento a frio retarda os processos fisiológicos como a respiração e a produção de calor vital, que levam à senescência das frutas. A redução da intensidade respiratória reduz as perdas de aroma, sabor, cor e outros atributos de qualidade do produto armazenado (FILGUEIRAS et al., 1996).

Carvalho \& Manica (1994), estudando a influência de estádios de maturação e condições de armazenamento da acerola, concluíram que frutos de acerolas verdes mantidos à temperatura ambiente deterioravam-se em seis ou sete dias, enquanto que quando em ambiente refrigerado $\left(5,5\right.$ a $\left.8,0^{\circ} \mathrm{C}\right)$, prolongaram sua vida pós-colheita em até 20 dias. Não há relatos de trabalho com reguladores em póscolheita na acerola, mas o efeito retardador da giberelina e da citocinina na evolução da coloração, devido ao atraso na degradação da clorofila, conforme o relatado por Chitarra \& Chitarra (1990) e Ludford (1995), não foi observado durante o período estudado. Lima \& Durigan (2002), utilizando reguladores vegetais em pós-colheita de goiabas 'Paluma', também não observaram efeito retardador da giberelina e da citocinina (devido ao atraso na degradação da clorofila), na evolução da coloração. Também a firmeza dos frutos não foi influenciada pelos reguladores vegetais.

O teor de sólidos solúveis ( ${ }^{\circ}$ Brix) tem sido usado como índice de maturidade para manga, laranja e outras. Conforme a Tabela 1, observa-se que os valores dos teores de sólidos solúveis variaram de 7,23 a 8,23 aproximando-se dos valores de Nogueira et al. (2002), que obtiveram valores que variaram de 7,07 a 7,80 para frutos verdes de acerolas. Horvitz et al. (2003) não encontraram diferenças significativas para essa característica em seu estudo. Entre os tratamentos não houve diferenças significativas em nenhuma das avaliações, o que mostra que os reguladores utilizados não interferiram nesta característica.

A acidez titulável (AT) encontrada para os frutos de acerola variou de 1,00 a 1,49 g de ác. málico100 g ${ }^{-1}$ de polpa (Tabela 1) não havendo diferença significativa entre os tratamentos. Esses valores aproximam-se dos valores encontrados por Alves (1993) que são de 1,65 no estádio verde de maturação.

$\mathrm{Na}$ análise de vitamina $\mathrm{C}$, os valores variaram de 1843,1 a $2559,9 \mathrm{mg} 100 \mathrm{~mL}^{-1}$ da amostra (Tabela 1), também não apresentando diferença significativa entre os tratamentos. Os valores no presente estudo concordam com os relatados por Alves (1993), que obteve valor de $1822 \mathrm{mg}$ $100 \mathrm{~g}^{-1}$ para frutos verdes no Estado de São Paulo e Kanno et al. (2000) em Junqueirópolis -SP de 2178,8 mg 100g-1 .

Carvalho \& Manica (1994) destacaram que frutos verdes de acerola mantidos sob refrigeração apresentaram vida de até 20 dias, apresentando teor de ácido ascórbico

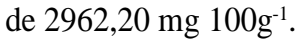

Nogueira et al. (2002), avaliando o conteúdo de vitamina $\mathrm{C}$, observaram decréscimo dos valores com a maturação dos frutos, sendo que os frutos verdes apresentaram valores superiores aos encontrados nos frutos maduros.

Neste trabalho não ocorreu redução do conteúdo de vitamina $\mathrm{C}$ em nenhum dos tratamentos, provavelmente, porque a refrigeração inibiu as reações oxidativas e retardou os processos fisiológicos, assim como os resultados obtidos por Antunes et al. (2003). Neste trabalho não foi verificada interação significativa para dias de armazenamento e doses de reguladores vegetais.

Através dos resultados obtidos e nas condições deste experimento, pode-se concluir que os reguladores vegetais não tiveram efeito no aumento da conservação refrigerada de acerolas verdes, sendo que somente a refrigeração foi suficiente para conservá-las durante 14 dias. 
TABELA 1 - Teor de sólidos solúveis ( ${ }^{\circ}$ Brix), acidez titulável (g ác.málico $100 \mathrm{~g}^{-1}$ de polpa) e vitamina $\mathrm{C}$ (mg ác. ascórbico $100 \mathrm{~mL}^{-1}$ ) de acerolas armazenadas durante 14 dias, a $8 \pm 1^{\circ} \mathrm{C}$, submetidas aos tratamentos com reguladores vegetais.

\begin{tabular}{|c|c|c|c|c|c|}
\hline \multirow{3}{*}{ Tratamentos } & \multicolumn{5}{|c|}{ Sólidos Solúveis } \\
\hline & \multicolumn{5}{|c|}{ Dias pós-colheita } \\
\hline & 2 & 6 & 10 & 14 & Médias \\
\hline Controle & $7,87 *$ & $8,03 *$ & $8,23 *$ & $7,67 *$ & 7,95 \\
\hline $\mathrm{GA}_{3} 50 \mathrm{mg} \mathrm{L}^{-1}$ & 8,00 & 7,80 & 7,53 & 7,63 & 7,74 \\
\hline $\mathrm{GA}_{3} 100 \mathrm{mg} \mathrm{L}^{-1}$ & 8,00 & 8,03 & 7,60 & 7,33 & 7,74 \\
\hline BAP $50 \mathrm{mg} \mathrm{L}^{-1}$ & 7,87 & 7,80 & 7,43 & 7,37 & 7,62 \\
\hline BAP $100 \mathrm{mg} \mathrm{L}^{-1}$ & 7,23 & 8,17 & 7,80 & 7,43 & 7,66 \\
\hline $\mathrm{CV}(\%)$ & 5,13 & 3,12 & 6,16 & 4,88 & - \\
\hline \multicolumn{6}{|c|}{ Acidez Titulável } \\
\hline \multirow{2}{*}{ Tratamentos } & \multicolumn{5}{|c|}{ Dias pós-colheita } \\
\hline & 2 & 6 & 10 & 14 & Médias \\
\hline Controle & $1,24^{*}$ & $1,38^{*}$ & $1,29 *$ & $1,15^{*}$ & 1,27 \\
\hline $\mathrm{GA}_{3} 50 \mathrm{mg} \mathrm{L}^{-1}$ & 1,23 & 1,17 & 1,03 & 1,29 & 1,18 \\
\hline $\mathrm{GA}_{3} 100 \mathrm{mg} \mathrm{L}^{-1}$ & 1,12 & 1,32 & 1,19 & 1,31 & 1,23 \\
\hline BAP $50 \mathrm{mg} \mathrm{L}^{-1}$ & 1,11 & 1,49 & 1,19 & 1,00 & 1,20 \\
\hline BAP $100 \mathrm{mg} \mathrm{L}^{-1}$ & 1,29 & 1,45 & 1,12 & 1,19 & 1,26 \\
\hline \multirow[t]{2}{*}{$\mathrm{CV}(\%)$} & 10,38 & 9,70 & 9,36 & 16,03 & - \\
\hline & \multicolumn{5}{|c|}{ Vitamina C } \\
\hline \multirow{2}{*}{ Tratamentos } & \multicolumn{5}{|c|}{ Dias pós-colheita } \\
\hline & 2 & 6 & 10 & 14 & Médias \\
\hline Controle & $2132,3^{*}$ & $2324,9 *$ & $2559,9^{*}$ & $2242,4^{*}$ & 2314,9 \\
\hline $\mathrm{GA}_{3} 50 \mathrm{mg} \mathrm{L}^{-1}$ & 2320,2 & 1990,7 & 2104,6 & 1941,5 & 2089,3 \\
\hline $\mathrm{GA}_{3} 100 \mathrm{mg} \mathrm{L}^{-1}$ & 2089,4 & 2260,3 & 2289,7 & 1921,9 & 2140,3 \\
\hline BAP $50 \mathrm{mg} \mathrm{L}^{-1}$ & 2337,7 & 2205,9 & 2101,5 & 1843,1 & 2122,1 \\
\hline BAP $100 \mathrm{mg} \mathrm{L}^{-1}$ & 2173,2 & 2104,5 & 2157,2 & 2250,0 & 2171,2 \\
\hline $\mathrm{CV}(\%)$ & 10,80 & 11,39 & 17,01 & 18,03 & - \\
\hline
\end{tabular}

* Médias não diferem significativamente entre si pelo teste $\mathrm{F}$.

\section{REFERÊNCIAS BIBLIOGRÁFICAS}

AHRENS, M. J.; BARMORE, C. R. Interative effects of washing, film wrapping and ethylene concentration on color development in grapefruit flavedo. Scientia Horticulturae, Amsterdam, v. 34, n. 3/4, p. 275-281, 1988.

ALVES, R. E. Acerola (Malpighia emarginata D.C.): fisiologia da maturação e armazenamento refrigerado sob atmosfera ambiente e modificada. 1993. 99 f. Dissertação (Mestrado em Fisiologia Vegetal) - Escola Superior de Agricultura de Lavras, Lavras, 1993.

ALVES, R. E. Características das frutas para exportação. In: GORGATI NETTO, A.; ARDITO, E. F. G.; GARCIA, E. E. C. (Eds.). Acerola para exportação: procedimentos de colheita e pós-colheita. Brasília, DF: Embrapa-SPI, 1996. p. 9-21. (Série Frupex, 21).

ALVES, R. E.; MENEZES, J. B.; SILVA, S. M. Colheita e pós-colheita da acerola. In: SIMPÓSIO BRASILEIRO SOBRE ACEROLA NO BRASIL, 1., 1995, Vitória da Conquista. Anais... Vitória da Conquista: Universidade Estadual do Sudoeste da Bahia, 1995. p. 77-89.

ANTUNES, L. E. C.; DUARTE FILHO, J.; SOUZA, C. M. Conservação pós-colheita de frutos de amoreira-preta. Pesquisa Agropecuária Brasileira, Brasília, v. 38, n. 3, p. 413-419, 2003.

BEZERRA NETO, E.; ANDRADE, A. G. de; BARRETO, L. $P$. Análise química de tecidos e produtos vegetais. Recife: UFRPE, $1994.80 \mathrm{p}$. 
CARVALHO, R. I. N.; MANICA, I. Influência de estádios de maturação e condições de armazenamento na conservação da acerola (Malpighia glabra L.). Pesquisa Agropecuária Brasileira, Brasília, v. 29, n. 5, p. 681-688, 1994.

CARVALHO, V. D. Qualidade e conservação pós-colheita de goiabas. Informe Agropecuário, Belo Horizonte, v. 17, n. 179 , p. 48-54, 1994.

CHITARRA, M. I. F.; CHITARRA, A. B. Pós-colheita de frutos e hortaliças: fisiologia e manuseio. Lavras: ESAL/ FAEPE, 1990. $320 \mathrm{p}$.

CRUZ, V. D.; D'ARCE, L. P. G.; CASTILHO, V. M. Variações no teor de ácido ascórbico de acerolas (M. glabra L.) em função do estágio de maturação e temperatura de estocagem. Arquivos de Biologia e Tecnologia, Curitiba, v. 38, n. 2, p. 331-337, 1995.

FILGUEIRAS, H. A. C.; CHITARRA, M. I. F.; CHITARRA, A. B. Armazenamento de ameixas sob refrigeração e atmosfera modificada 2: colapso interno (internal breakdown) e textura. Revista Brasileira de Fruticultura, Cruz das Almas, v. 18, n. 1, p. 129-135, 1996.

FIORAVANÇO, J. C.; MANICA, I.; PAIVA, M. C. Uso de citocinina e recobrimentos em limão 'Tahiti' armazenado em temperatura controlada. Pesquisa Agropecuária Brasileira, Brasília, v. 30, n. 1, p. 81-87, 1995.

HORVITZ, S.; GODOY, C.; CAMELO, A. F. L.; YOMMI, A. Application of gibberellic acid to sweet cherries (Prunus avium) cv. 'Sweetheart': effects on fruit quality at harvest and during cold storage. Acta Horticulturae, Wageningen, v. 628, n. 1, p. 311-316, 2003.

INSTITUTO BRASILEIRO DE FRUTAS. Soluções fruta a fruta: acerola. São Paulo, 1995. 59 p.
KANNO, O. Y.; RIZZI, C.; KAVATI, R. Acerola olivier. In: . Novas variedades brasileiras de frutas. Jaboticabal: Sociedade Brasileira de Fruticultura, 2000. p. 24-25.

LIMA, M. A.; DURIGAN, J. F. Reguladores vegetais na conservação pós-colheita de goiabas 'Paluma'. Revista Brasileira de Fruticultura, Jaboticabal, v. 24, n. 2, p. 232236, 2002.

LUDFORD, P. L. Postharvest hormone changes in vegetables and fruits. In: DAVIES, P. J. (Ed.). Plant hormones. The Netherlands: Kluwer Academic, 1995. p. 725-750.

MELO, E. A.; LIMA, V. L. A. G.; NASCIMENTO, P. P. Temperatura no armazenamento de pitanga. Scientia Agricola, Piracicaba, v. 57, n. 4, p. 629-634, 2000.

NOGUEIRA, R. J. M. C.; MORAES, J. A. P. V.; BURITY, H. A.; SILVA JÚNIOR, J. F. Efeito do estádio de maturação dos frutos nas características físico-químicas de acerola. Pesquisa Agropecuária Brasileira, Brasília, v. 37, n. 4, p. 463-470, 2002.

PETINARI, R. A.; TARSITANO, M. A. A. Análise econômica da produção de acerola para mesa, em Jales, SP: um estudo de caso. Revista Brasileira de Fruticultura, Jaboticabal, v. 24, n. 2, p. 411-415, 2002.

PÍPOLO, V. C.; PRETE, C. E. C.; GONZALEZ, M. G. N.; POPPER, I. O. Novas cultivares de acerola (Malpighia emarginata DC): UEL3- Dominga, UEL4- Lígia e UEL5Natália. Revista Brasileira de Fruticultura, Jaboticabal, v. 24, n. 1, p. 124-126, 2002.

TEAOTIA, S. S.; TRIPATH, C. S.; SINGH, R. N. Effect of growth substances on ripening and quality of guava (Psidium guajava L.). Journal Food Science Technology, Chicago, n. 9, p. 38, 1972. 